\title{
Beyond Analog Gravity: The Case of Exceptional Dynamics
}

\author{
M. Novello* and E.Goulart ${ }^{\dagger}$ \\ Instituto de Cosmologia Relatividade Astrofisica ICRA - CBPF \\ Rua Dr. Xavier Sigaud, 150, CEP 22290-180, Rio de Janeiro, Brazil
}

(Dated: November 5, 2018)

\begin{abstract}
We show that it is possible to go beyond the propagation aspects usually contemplated in the analog models of gravity. Until this moment, the emergence of a metric appears in the description of excitations around a given background solution or in the study of field discontinuities in the geometrical optics regime. We now overcome some limitations of the above analogies and exhibit the form of a Lagrangian that describes the dynamics of a self-interacting field $\varphi$ as an interaction between $\varphi$ and its associated effective metric $\widehat{g}^{\mu \nu}$. In other words the non-linear equation of motion of the field may be interpreted as the gravitational influence on $\varphi$ by its own effective metric which, in our scheme becomes an active partner of the dynamics of $\varphi$.
\end{abstract}

PACS numbers:

\section{INTRODUCTION}

In recent years, intense activity on analogue gravity has been developed [1].This was concentrated in nonlinear electrodynamics, acoustics, hydrodynamics, optics inside media and various condensed matter systems. This term (analog gravity) implies the description of distinct physical processes as modifications of the metrical structure of the background space-time. Until now this analogy had been limited to perturbative aspects restricting it to the propagation of excitations (photons and quasiparticles) through a medium or a background field configuration. In this paper we give the first example which goes beyond this limitation describing dynamical features of fields in terms of their respective effective metric ${ }^{1}$. The main steps to achieve this result are the following:

- Consider a non linear field theory described in a flat Minkowski background;

- Note that the propagation of the discontinuities of the field yields the irruption of a second metric $\widehat{g}^{\mu \nu}$ such that the path of the waves are null geodesics in this effective geometry;

- There exists a special class of theories such that its corresponding dynamics (which we will deserve the name exceptional) can be described alternatively as the gravitational interaction of the field in a given curved geometry;

- The geometry of such gravitational space-time is precisely the effective metric $\widehat{g}^{\mu \nu}$.

Thus the claim of the present paper is that the selfinteraction described by exceptional dynamics is de-

\footnotetext{
*M. Novello is Cesare Lattes ICRANet Professor: novello@cbpf.br †egoulart@cbpf.br

1 We would like to stress that this dynamical aspect we are investigating has nothing to do with mimicking Einstein's equations through effective metrics.
}

scribed in an equivalent way as the gravitational interaction of the field with its own effective metric.

For pedagogical reasons we limit our analysis here to the case of non linear scalar fields. Generalization to the case of other fields, like non-linear Electrodynamics will be described elsewhere.

\section{THE KINEMATICAL ANALOGY}

Let us give a simple example. Consider a scalar field $\varphi$ propagating in a flat spacetime whose dynamics is provided by the non-linear Lagrangian [2],

$$
L=L(w)
$$

where

$$
w \equiv \partial_{\mu} \varphi \partial_{\nu} \varphi \eta^{\mu \nu}
$$

is the canonical kinetical term. The equation of motion for $\varphi$ reads:

$$
\partial_{\mu}\left(L_{w} \partial_{\nu} \varphi \eta^{\mu \nu}\right)=0
$$

where $L_{w}$ denotes the first derivative of $L$ with respect to $w$. By derivating the left hand side we obtain the explicit form

$$
L_{w} \square \varphi+2 L_{w w} \partial^{\mu} \varphi \partial^{\nu} \varphi \partial_{\mu} \partial_{\nu} \varphi=0,
$$

with $\square \equiv \eta^{\mu \nu} \partial_{\mu} \partial_{\nu}$. This constitutes a quasi-linear second order partial differential equation for $\varphi$. We are interested in evaluating the characteristic surfaces of wave propagation in this theory. The most direct and elegant way to pursue this goal is to use the Hadamard formalism [3].

Let $\Sigma$ be a surface of discontinuity of the scalar field $\varphi$. The discontinuity of an arbitrary function $f$ is given by:

$$
[f(x)]_{\Sigma}=\lim _{\epsilon \rightarrow 0^{+}}(f(x+\epsilon)-f(x-\epsilon))
$$


The field $\varphi$ and its first derivative $\partial_{\mu} \varphi$ are continuous across $\Sigma$, while the second derivative presents a discontinuity:

$$
\begin{aligned}
{[\varphi]_{\Sigma} } & =0, \\
{\left[\partial_{\mu} \varphi\right]_{\Sigma} } & =0, \\
{\left[\partial_{\mu} \partial_{\nu} \varphi\right]_{\Sigma} } & =k_{\mu} k_{\nu} \xi(x),
\end{aligned}
$$

where $k_{\mu}:=\partial_{\mu} \Sigma$ is the propagation vector and $\xi(x)$ the amplitude of the discontinuity. From the above conditions we obtain that both $L_{w}$ and $L_{w w}$ are continuous functions across $\Sigma$. Using these discontinuity properties in the equation of motion (2) it follows that only the second order derivative terms contribute. We obtain the relation

$$
L_{w} \eta^{\mu \nu}\left[\partial_{\mu} \partial_{\nu} \varphi\right]_{\Sigma}+2 L_{w w} \partial^{\mu} \varphi \partial^{\nu} \varphi\left[\partial_{\mu} \partial_{\nu} \varphi\right]_{\Sigma}=0 .
$$

Thus, using Hadamard conditions it follows

$$
k_{\mu} k_{\nu}\left(L_{w} \eta^{\mu \nu}+2 L_{w w} \partial^{\mu} \varphi \partial^{\nu} \varphi\right)=0,
$$

This equation suggests the introduction of the effective metric defined by:

$$
\widehat{g}^{\mu \nu}:=L_{w} \eta^{\mu \nu}+2 L_{w w} \partial^{\mu} \varphi \partial^{\nu} \varphi
$$

Thus there are two distinct metrics in this framework: the Minkowskian $\eta^{\mu \nu}$ that enters in the dynamics of the field $\varphi$ and the effective metric $\widehat{g}^{\mu \nu}$ that controls the propagation of the waves. Note that, once the vector of discontinuity $k_{\mu}$ is a gradient, discontinuities of the field $\varphi$ propagate through null geodesics in the effective metric $\widehat{g}_{\mu \nu}$, i.e.

$$
\hat{g}^{\mu \nu} k_{\alpha ; \mu} k_{\nu}=0,
$$

where ";" stands for the covariant derivative evaluated with the effective metric. The inverse $\widehat{g}_{\mu \nu}$ of (8) is obtained through the condition $\hat{g}^{\mu \alpha} \hat{g}_{\alpha \nu}=\delta_{\nu}^{\mu}$ :

$$
\widehat{g}_{\mu \nu}=\frac{1}{L_{w}} \eta_{\mu \nu}-\frac{2 L_{w w}}{L_{w} \Psi} \partial_{\mu} \varphi \partial_{\nu} \varphi,
$$

where we defined $\Psi:=L_{w}+2 w L_{w w}$. For latter reference we note that the determinant of a mixed tensor $\mathbf{T}=T_{\beta}^{\alpha}$ may be expressed in terms of traces of its powers in the form

$$
\begin{aligned}
-4 \operatorname{det} \mathbf{T} & =\operatorname{Tr}\left(\mathbf{T}^{4}\right)-\frac{4}{3} \operatorname{Tr}(\mathbf{T}) \operatorname{Tr}\left(\mathbf{T}^{3}\right) \\
& -\frac{1}{2}\left(\operatorname{Tr}\left(\mathbf{T}^{2}\right)\right)^{2}+(\operatorname{Tr}(\mathbf{T}))^{2} \operatorname{Tr}\left(\mathbf{T}^{2}\right) \\
& -\frac{1}{6}(\operatorname{Tr}(\mathbf{T}))^{4} .
\end{aligned}
$$

This is an immediate consequence of the CayleyHamilton theorem. Applying this formula to the effective metric (8) one obtains, after a straightforward calculation

$$
\sqrt{-\widehat{g}}=L_{w}^{-2}(1+\beta w)^{-1 / 2},
$$

where we have defined $\beta=2 L_{w w} / L_{w}$. Note that, the square-root of the determinant is real only if the condition

$$
1+2 w L_{w w} / L_{w}>0
$$

is satisfied. This is the same as to guarantee the hiperbolicity of the equations of motion and henceforth the existence of waves. Nevertheless, we remark that the effective metric that controls the propagation of these waves is not unique and is determined up to a conformal factor. However, as it occurs in typical non-linear theory, the dynamics is not conformal-invariant.

\section{EXCEPTIONAL DYNAMICS}

Let us note that the discontinuities of the field propagate in a curved space-time, although the field $\varphi$ is described by a non-linear theory in Minkowski geometry [4].

In the framework of general relativity it is the presence of gravity that allows the existence of curvature in the geometry. This has led to the interpretation of the dispersion relation of non-linear fields in terms of an effective metric as nothing but the simplification of its description, that is, a matter of language.

At this point we face the following question: is it be possible that among all non-linear theories one can select a special class such that the dynamics of the field itself is described in terms of the effective metric? That is, is it be possible to unify the dynamics of the field with the propagation of its waves such that just one metric appeared? ${ }^{2}$

If this is possible, then we are in the presence of a field that mimics a kind of gravitational interaction (coupling) by means of its own equation of motion. In other words, the equation of evolution of $\varphi$ is equivalent to the gravitational interaction between $\varphi$ and its effective metric. This means that the equation of motion (11) can be written under the equivalent form

$$
\widehat{\square} \varphi \equiv \frac{1}{\sqrt{-\hat{g}}} \partial_{\mu}\left(\sqrt{-\hat{g}} \partial_{\nu} \varphi \hat{g}^{\mu \nu}\right)=0 .
$$

In general it is not possible to re-write the nonlinear equation (11) in the above form for an arbitrary lagrangian. However, we will show next that there exist some special situations where this implementation becomes feasible. We first note the following relation

$$
\partial_{\nu} \varphi \hat{g}^{\mu \nu}=\left(L_{w}+2 w L_{w w}\right) \partial_{\nu} \varphi \eta^{\mu \nu}
$$

\footnotetext{
${ }^{2}$ Note that this is not equivalent to the known property - that occurs in hydrodynamics (and also in field theory) - that the characteristic propagation of the field coincides with the characteristic propagation of its perturbations (see appendix for a detailed discussion).
} 
It then follows that the dynamics described by (1) and (15) will be the same, provided the Lagrangian satisfies the condition

$$
L_{w}=\sqrt{-\hat{g}}\left(L_{w}+2 w L_{w w}\right) .
$$

Using the expression for the determinant (13) the equivalence is provided by the nonlinear differential equation for the lagrangian

$$
2 w L_{w w}+L_{w}-L_{w}^{5}=0 .
$$

We will call the system described by Lagrangians that satisfies condition (18) as Exceptional Dynamics. In other words, non-linear systems described by exceptional dynamics may be alternatively interpreted as fields gravitationally coupled to its own effective geometry. Thus the field and the corresponding waves agree in the interpretation that the geometry of the space-time is given by $\widehat{g}_{\mu \nu}$.

Equation (18) is such that the first derivative of the lagrangian with respect to $w$ may be obtained explicitly. Indeed, one obtains that

$$
L_{w}= \pm \frac{1}{\left(1-\lambda w^{2}\right)^{\frac{1}{4}}}
$$

where $\lambda$ is an arbitrary real positive constant. The general solution of equation (18) may be obtained as an infinite series given by the hypergeometric function

$$
L(w)= \pm w H y p 2 F 1\left[\frac{1}{2}, \frac{1}{4}, \frac{3}{2}, \lambda w^{2}\right] .
$$

We note that this dynamics is such that the admissible values of $w$ are restricted to the domain $w^{2}<\lambda$. Thus, the theory naturally avoids arbitrarily large values of the kinematical term. Second, the lagrangian is a monotonic function of $w$ as an immediate consequence of equation (19). Third, the theory admits the linear theory as a limiting case for small values of $w$, as we will soon see. Lastly, the resulting function is odd, i.e $L(w)=L(-w)$.

A comparison between the exceptional lagrangian (20) and the canonical linear lagrangian $L=w$ is given in figure I. From the plot we see that, in fact, the new theory is described by a simple function that possess many desirable features. We filled the space between the functions to give a concrete idea of how they differ from each other in this domain. Note that, although the maximum difference happens in the boundaries $w^{2}=\lambda$, the exceptional lagrangian is very similar to the linear one for small values of " $w$ ".

It is instructive to expand the exceptional lagrangian to obtain a clear idea of its behavior in terms of the parameter $\lambda$. We obtain

$$
L(w)=w+\frac{\lambda}{12} w^{3}+\frac{\lambda^{2}}{32} w^{5}+\frac{15 \lambda^{3}}{896} w^{7}+O[x]^{9}
$$

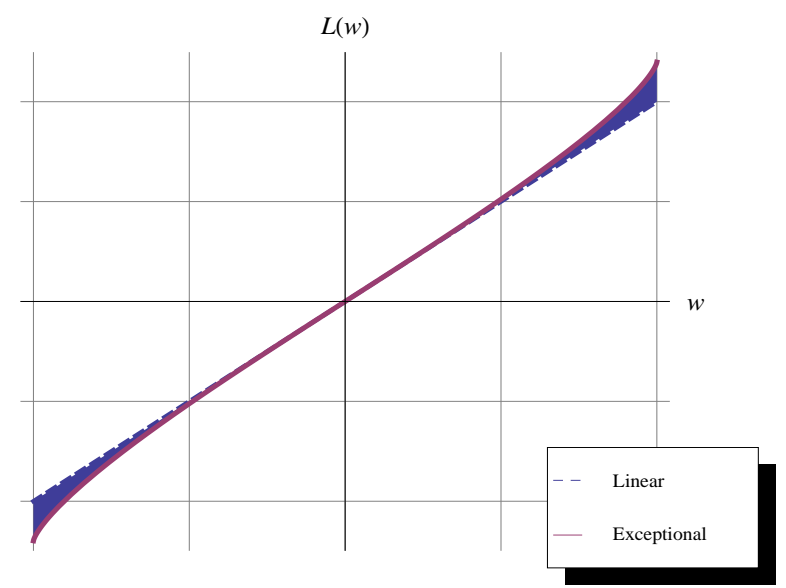

FIG. 1:

Comparison between linear and exceptional lagrangians.

Note that for small values of the constant, $\lambda<<1$ the exceptional dynamics reduces to a cubic lagrangian ${ }^{3}$. The linear theory is obviously recovered when $\lambda=0$, implying that both excitations (discontinuities) and field dynamics are described by the same effective metric i.e. $\eta^{\mu \nu}$.

\section{ANOTHER SIMPLE EXAMPLE}

In the precedent section we examined the case in which a self-interacting scalar field generates a geometrical arena for its own propagation. The presence of an effective metric that controls the propagation of the discontinuities of the field can be extended in such a way that the original theory, describing the dynamics of the scalar field in a flat Minkowski arena can be alternatively described as the gravitational interaction of the field. Then we have shown a non-expected result: the metric field that describes this gravitational effect is nothing but the same effective metric that controls the wave propagation.

In other words, our main achievement was to produce a scenario where both the propagation of the field discontinuities and the field dynamics are controlled by the same metric structure. The nonlinearities are such that the equations in Minkowski space-time mimic the dynamics of a "free-field" embedded in a curved space-time generated by the field itself.

Let us now provide another simple example of a physical system where the exceptional dynamics may be relevant to real practical situations and may be used to

\footnotetext{
${ }^{3}$ We could arrive at this result assuming from the beginning a lagrangian in the vicinity of the linear theory of the form $L(w)=$ $w+\epsilon f(w)$ with $\epsilon^{2}<<\epsilon$ and solving the simplified equation $w f_{w w}-2 f_{w}=0$.
} 
investigate dynamical aspects of fields in curved spacetimes. The basic system consists of Maxwell's electrodynamics inside (linear) dielectric medium in motion. The quantities we will deal with consists in tensors $F_{\mu \nu}$ and $P_{\mu \nu}$ and a normalized time-like vector field $v^{\mu}(x)$ that is $\eta_{\mu \nu} v^{\mu} v^{\nu}=1$ which represents the velocity field of the material. We have

$$
\begin{aligned}
& F_{\mu \nu}=E_{\mu} v_{\nu}-E_{\nu} v_{\mu}+\eta_{\mu \nu}{ }^{\alpha \beta} v_{\alpha} B_{\beta} \\
& P_{\mu \nu}=D_{\mu} v_{\nu}-D_{\nu} v_{\mu}+\eta_{\mu \nu}{ }^{\alpha \beta} v_{\alpha} H_{\beta}
\end{aligned}
$$

We restrict our analysis to the case of isotropic constitutive laws provided by

$$
D_{\alpha}=\epsilon(x) E_{\alpha} \quad B_{\alpha}=\mu(x) H_{\alpha} .
$$

The equations of motion are

$$
P_{, \nu}^{\mu \nu}=0 \quad F_{[\mu \nu, \alpha]}=0,
$$

and the study of field discontinuities inside such material leads to Gordon's metric

$$
\hat{g}^{\mu \nu}=\eta^{\mu \nu}+(\mu \epsilon-1) v^{\mu} v^{\nu} .
$$

Thus, the wave fronts inside an isotropic, heterogeneous and linear material is described by a null vector $k_{\mu}$ with respect to the effective metric i.e. $\hat{g}^{\mu \nu} k_{\mu} k_{\nu}=0$. Furthermore, it is immediate to show that $k_{\mu}$ is a geodesic with respect to $\hat{g}_{\mu \nu}$. This result is well known and was obtained several times in the literature of analog models, enabling the study of kinematical aspects of fields in the presence of gravitation. On the other hand, there exist various situations, typical of certain class of materials, where it is possible to go beyond this kinematical analogy. In these cases the field dynamics itself is described by its dependence on the effective metric, as in the case of exceptional dynamics.

In fact, using the determinant formula (11) it follows that

$$
\hat{g} \equiv \operatorname{det}\left(\hat{g}_{\mu \nu}\right)=-\frac{1}{\mu \epsilon} .
$$

Also, from the definition of the effective metric we obtain the identity

$$
\hat{F}^{\mu \nu} \equiv \hat{g}^{\mu \alpha} \hat{g}^{\nu \beta} F_{\alpha \beta}=\mu P^{\mu \nu}
$$

Using (24) and (25) simultaneously it is possible to write the first of Maxwell equations (23) in the form

$$
\left(\frac{1}{\mu} \hat{F}^{\mu \nu}\right)_{, \nu}=0 .
$$

In the case of impedance matched materials where the ratio $\epsilon / \mu$ is constant, we can re-write this equation in the very suggestive form

$$
\left(\sqrt{-\hat{g}} \hat{F}^{\mu \nu}\right)_{, \nu}=0
$$

Finally the complete set of Maxwell equations (23) in this medium can be written as

$$
\hat{\nabla}_{\nu} \hat{F}^{\mu \nu}=0 \quad \hat{\nabla}_{[\alpha} \hat{F}_{\mu \nu]}=0,
$$

where $\hat{\nabla}_{\alpha}$ is the covariant derivative written in terms of $\hat{g}_{\mu \nu}$. This means that the effective metric, that describes the characteristics, has an active part in the very description of the field dynamics. This situation can be used as a tool to investigate simultaneously kinematical and dynamical aspects of fields interacting with gravity in laboratories.

\section{CONCLUSION}

Let us summarize the novelty of our analysis in a broad sense:

- For any field theory described on a Minkowski background by a non-linear Lagrangian $L=L(w)$ the discontinuity of $\varphi$ propagates as null geodesics in an effective metric $\widehat{g}^{\mu \nu}$;

- As such, the theory presents a duplicity of metrics: the field is described in flat Minkowski spacetime and its corresponding waves propagate as null geodesics in a curved geometry;

- It is possible to unify the description of the dynamics of $\varphi$ in such a way that only one metric appears. This is possible for those Lagrangians that represent exceptional dynamics;

- In this case, the self-interaction of $\varphi$ is described equivalently as if it were interacting minimally with its own effective geometry, allowing the interpretation in terms of an emergent gravitational phenomenon.

The structure of the nonlinear equations of motion suggests that the previous procedure can be adapted to other structures like non linear electrodynamics in a moving dielectric, vector and tensor field theories. We will develop this idea in another paper.

\section{APPENDIX}

In this appendix we would like to emphasize that the possibility to describe the dynamics of scalar field as an interaction with its own effective metric — and as such, mimic gravitational interaction - is not valid for all types of non-linear theory but only for a specific class - which we called exceptional dynamics.

On the other hand, one should not confuse such property with a generic one concerning the propagation of 
the discontinuities. Indeed, the condition of a given Lagrangian to describe an exceptional dynamics is completely independent of the known fact that the characteristic propagation of the field is similar to the characteristic propagation of its (linear) perturbations. That is both discontinuities are guided by the same effective metric $\hat{g}_{\mu \nu}$. Let us prove this.

The characteristic surfaces of the nonlinear equation of motion (11) are obtained by the analysis of its discontinuities. As we shown in a precedent section these surfaces are such that

$$
\hat{g}^{\mu \nu} k_{\mu} k_{\nu}=0
$$

with $\hat{g}^{\mu \nu}$ given by (8). We now turn to a diferent question. Let us consider a perturbation of the field $\varphi$, i.e.

$$
\varphi \rightarrow \varphi+\delta \varphi
$$

If we linearize equation (11) around a given background solution $\varphi$, retaining only first order terms in $\delta \varphi$, then what are the characteristic surfaces for the perturbation $\delta \varphi$ ? The linearized equations reads

$$
\begin{array}{ll} 
& \left(L_{w} \eta^{\mu \nu}+2 L_{w w} \varphi^{, \mu} \varphi^{, \nu}\right) \delta \varphi, \mu, \nu \\
+\quad & 2 L_{w w}\left(\square \varphi \varphi^{, \mu}+2 \varphi^{, \mu}{ }_{, \nu} \varphi^{, \nu}\right) \delta \varphi_{, \mu}+ \\
+\quad & 4 L_{w w w} \varphi, \alpha, \nu \\
& \varphi^{\alpha} \varphi^{, \nu} \varphi^{, \mu} \delta \varphi_{, \mu}=0
\end{array}
$$

Applying Hadamard's analysis to this new equation i.e.

$$
\begin{aligned}
{[\delta \varphi]_{\Sigma} } & =0 \\
{\left[\partial_{\mu} \delta \varphi\right]_{\Sigma} } & =0 \\
{\left[\partial_{\mu} \partial_{\nu} \delta \varphi\right]_{\Sigma} } & =k_{\mu} k_{\nu} \xi(x),
\end{aligned}
$$

we obtain the well-known result that the perturbations $\delta \varphi$ are governed by the same effective metric. Thus, the characteristic propagation of the field is equivalent to the characteristic propagation of its own disturbances. This fact is valid for any theory and is totally independent of the specification of the lagrangian.

\section{ACKNOWLEDGEMENTS}

MN would like to thank FINEP, CNPq and FAPERJ and EG FAPERJ for their financial support.
[1] A complete review on different applications of the method of the effective geometry was given in the workshop Artificial Black Holes, ed. M Novello, M. Visser and G. Volovik, World Scientific, 2002.

[2] L. J. Hall, J. Lykken, and S. Weinberg, Phys. Rev. D 27, 2359 (1983); E. Cremmer, S. Ferrara, L. Girardello, and A. Van Proeyen, Nucl. Phys. B 212, 413 (1983); S. K. Soni and H. A. Weldon, Phys. Lett. B 126, 215 (1983).

L. P. Chimento, M. Forte, and R. Lazkoz, astro-ph/0407288; L. P. Chimento, Phys. Rev. D 69, 123517 (2004).

M Novello, M Makler, L S Werneck and Romero,C.A.: Phys. Rev. D71, 043515 (2005).

D. Kutasov, M. Marino, and G. W. Moore, JHEP 0010, 045 (2000); K. Li and E. Witten, Phys. Rev. D 48, 853 (1993); A. Sen, JHEP 9910, 008 (1999).

N. Arkani-Hamed, H.-Ch. Cheng, M. A. Luty, and S. Mukohyama, JHEP 05, 074 (2004).

C. Armendariz-Picon, T. Damour, and V. F. Mukhanov, Phys. Lett. B 458, 209 (1999).

J. Garriga and V. F. Mukhanov, Phys. Lett. B 458, 219 (1999).

[3] Y. Choquet-Bruhat, C. de Witt-Morette, and M. DillardBleick, Analysis, Manifolds and Physics, North-Holland, New York, 1977, p. 455; see also J. Hadamard, Leçons sur la propagation des ondes et les équations de l'hydrodynamique, Hermann, Paris, 1903.

M. Novello, V. De Lorenci, J. Salim, and R. Klippert, Physical Review D 61, 45001 (2000) and references therein.

M. Novello, S. E. Perez Bergliaffa and Salim,J.: Phys. Rev.
D69, 127301 (2004).

M. Novello, V. A. De Lorenci, J. M. Salim, and R. Klippert, Class. Quantum Gravity 20, 859 (2003).

M. Novello and S. E. Perez Bergliaffa, in Proceedings of the Xth Brazilian School of Gravitation and Cosmology, M. Novello and S. E. Perez Bergliaffa eds., AIP Conf. Proc. 668, 288 (2003), gr-qc/0302052.

[4] Novello, M. and Salim,J.M. in Phys. Rev. D63, 083511, (2001).

A complete list of references can be found in C. Barcelo, S. Liberati, and M. Visser, Classical and Quantum Gravity 18, 3595 (2001).

W. Unruh, Phys. Rev. Lett. 46, 1351 (1981), and Phys. Rev. D 51, 2827 (1995).

V. A. De Lorenci, Renato Klippert, M. Novello, J.M. Salim, Phys. Lett. B482, 134 (2000).

F. Baldovin, M. Novello, S.E. Perez Bergliaffa, and J.M. Salim, Class. Quant. Grav. 17, 3265 (2000).

M. Novello, J. M. Salim, V. A. De Lorenci, and E. Elbaz, Phys. Rev. D 63, 103516 (2001).

Bi-refringence versus bi-metricity, M. Visser, C. Barcelo, and S. Liberati. Contribution to the Festschrift in honor of Mario Novello (2003). gr-qc/0204017.

T. Jacobson and G. Kang, Classical and quantum Gravity 10, L201 (1993).

M. Novello, S.E. Perez Bergliaffa, J.M. Salim, Class. Quant. Grav. 17, 3821 (2000).

E. Goulart and SEP Bergliaffa in Class Quantum Gravity 26, 2009 (135015) 